\title{
Clinical Feasibility of Soft-Tissue Matching in Cone-Beam Computed Tomography (CBCT) Guidance for Liver Cancer Patients
}

\author{
Shengchao Jiao ${ }^{1}$, Jianrong $\mathrm{Dai}^{2}$, Weihu Wang ${ }^{2}$, Kuo $\mathrm{Men}^{2}$, Minghui $\mathrm{Li}^{2}$, Guishan $\mathrm{Fu}^{2}$, $\mathrm{Nan}^{2}{ }^{2}$, \\ Yexiong $\mathrm{Li}^{2}$ \& Yufeng Cheng ${ }^{1}$ \\ ${ }^{1}$ Department of Radiation Oncology, Qilu Hospital of Shandong University, Shandong University, Shandong, \\ China \\ ${ }^{2}$ Department of Radiation Oncology, Cancer Hospital (Institute), Chinese Academy of Medical Sciences, Peking \\ Union Medical College, Beijing, China \\ Correspondence: Jianrong Dai, Department of Radiation Oncology, Cancer Hospital (Institute), Chinese \\ Academy of Medical Sciences, Peking Union Medical College, Beijing, China. E-mail: \\ jianrong_dai@yahoo.com
}

Received: April 7, 2013

Accepted: May 14, 2013 Online Published: July 1, 2013

doi:10.5539/apr.v5n4p16

URL: http://dx.doi.org/10.5539/apr.v5n4p16

\begin{abstract}
The feasibility of soft-tissue matching in cone-beam computed tomography (CBCT) guidance for liver cancer patients is investigated and its equivalence to fiducial-marker matching on determining patient setup corrections has been studied. On the Elekta Synergy machine, daily CBCT volumetric images were acquired after setup with silver rings (SRs) in liver. The SRs were served as fiducial markers in our study. The CBCT images without SRs in liver were then obtained by digitally removing the SRs in each projection image using photoshop CS4.Considering fiducial-marker matching as a standard, the patient setup errors were compared between two two matching methods. A total of 90 datasets of volumetric images for 10 patients were used in the comparison. Pearson coefficient of correlation for the setup errors was as follows: $\mathrm{R}^{2}=0.718,0.724,0.785$ in the LR, AP and SI directions, respectively. A Bland-Altman analysis showed no significant trends. The percentage of errors within a $\pm 3 \mathrm{~mm}$ tolerance was respectively $95.56 \%, 95.83 \%, 91.11 \%$. The p-value (paired permutation test) are respectively $0.1217,0.919$ and 0.7685 in the three cardinal directions and are all greater than 0.05 . So, the null hypothesis cannot be refused, that is to say. Two methods have no significant difference. CBCT guidance using soft-tissue is an equivalent method for on-line image-guided radiotherapy for liver-cancer patients when compared to CBCT guidance using fiducial markers. It is feasible to implement $\mathrm{CBCT}$ image guidance using soft-tissue matching method clinically.
\end{abstract}

Keywords: cone-beam CT, fiducial markers, silver rings (SRs), Pearson coefficient, Bland-Altman analysis

\section{Introduction}

Cone-beam computed tomography (CBCT) has been used to correct setup errors for patients with liver cancer in many institutes. However, liver tumors are hard to distinguish on CBCT images since their densities are similar. Therefore, a surrogate for the tumor must be adopted for image guide radiation therapy (IGRT) of liver cancer. Fiducial markers, which have high density and a physical size, generally serve as surrogates to provide visibility of liver tumors in portal images. However, this method could induce substantial star artifacts in CT and CBCT images, which can reduce accuracy of setup corrections based on fiducial-markers matching and interfere with dose calculation and assessment .(Wunderink et al., 2008; Owen et al., 2008).

Wunderink et al. (2010) found that if fiducial markers cannot be implanted surrounding the tumor, marker guidance may induce set-up errors comparable to or greater than those of conventional set-up methods, as substantial rotations and deformations in marker groups were observed (Wunderink et al., 2010). Target coverage could then be jeopardized by marker guidance. In this study, hypothesizing that fiducial markers were closely surrounding the tumor tissue and the fiducial-marker matching served as the ground truth, we investigated the feasibility of soft-tissue matching in Cone-beam computed tomography (CBCT) guidance for liver cancer patients through checking its equivalence to fiducial-marker matching on determining patient setup corrections and this study is a retrospective study. 
Two different matching methods were adopted to acquire information of daily setup correction during an entire treatment course: (1) fiducial-marker matching (CBCT-SRs matching); (2) soft-tissue matching (CBCT-ST matching), namely, CBCT without sliver rings. Data of daily setup correction based on this two matching methods was compared and the level of correlation were also reported.

\section{Materials and Methods}

\subsection{Simulation and Planning}

Ten patients who need radiotherapy after their liver-cancer operation were involved in this study. Each patient had four sliver rings ( $1 \mathrm{~mm}$ height, and $4 \mathrm{~mm}$ in diameter) implanted in their livers during liver-cancer operation. For 3D external beam radiotherapy, they were immobilized with MedTech body frame and thermoplastic body mask. 4-Dimensional Average computed tomography (4D-Avg CT) images were acquired for treatment planning using a 24-slice computed tomography (CT) simulator (Bigbore, Philips) with a slice thickness of $3 \mathrm{~mm}$. Treatment plans were designed with the Pinnacle treatment planning system, version 8.0 (Philips/ADAC, Milpitas, CA). All patients were treated on a linear accelerator with kV imaging system (Synergy, Elekta Oncology Systems, Crawley). The geometric accuracy of the Synergy system had been discussed before (Sharpe et al., 2006; Jaffray et al., 2002).

\subsection{Image Acquisition and Process}

\subsubsection{CBCT-Srs Image Acquisition}

CBCT system can capture approximately 630 2-D projections through the $360^{\circ}$ gantry rotation in 2 minutes. $\mathrm{X}$-Ray Volume Imaging (XVI) reconstructs all projections to form a set of CBCT images. The reconstruction algorithm was based on Feldkamp's back projection algorithm (Feldkamp et al., 1984).

\subsubsection{CBCT-ST Image Acquisition}

The SRs in the liver produce artifacts in the reconstructed CBCT image sets. In order to get the CBCT images without SRs, the following procedure was implemented: a). Transform each projection image from Hospital Information System (HIS) format to Digital imaging and Communications in Medicine (DICOM) format with a self-developed program; b). SRs in the transformed projection image were erased by Spot healing brush tool in Photoshop CS4. The result for picking up this software is that it could reconstructed the pixel where SRs local by selecting similar pixel nearby the border of SRs (Figure 1); c). Transform back each projection image in HIS. Format and re-imported them to XVI; d). Reconstruct the processed CBCT dataset and get CBCT image without the SRs.

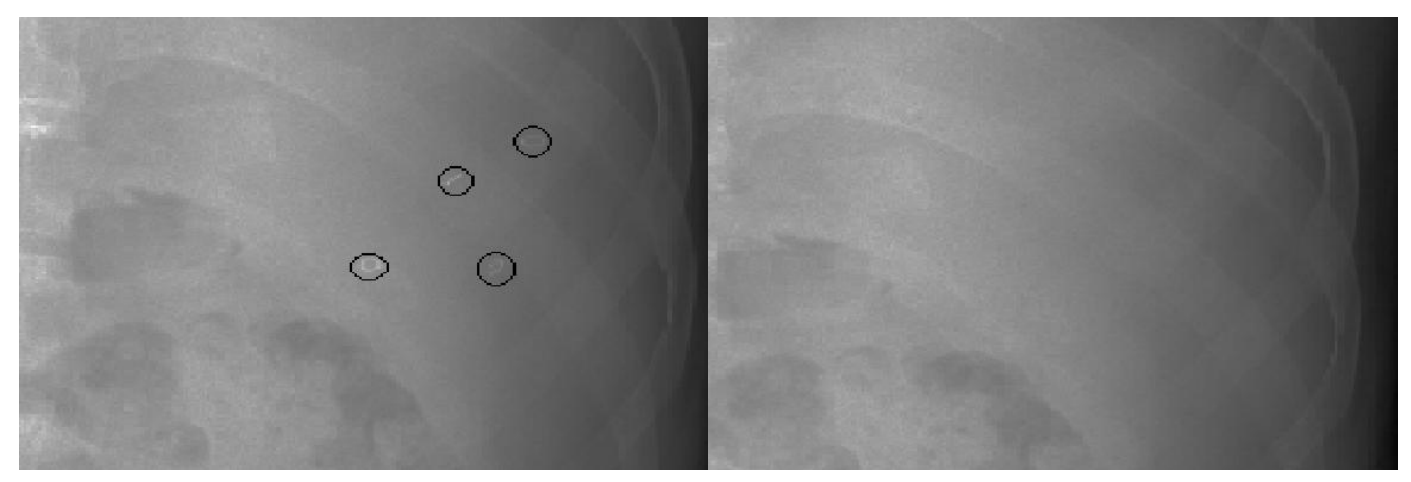

Figure 1. Projection image before (upper panel) and after (down panel) the SRs were removed (the black circles represent the position of SRs)

\subsubsection{D-Avg CT Reference Image without SRs Acquisition}

4D-Avg CT images are exported from XVI and processed using the same method mentioned above and 4D-Avg CT image for reference without SRs are achieved at last (Figure 2). 


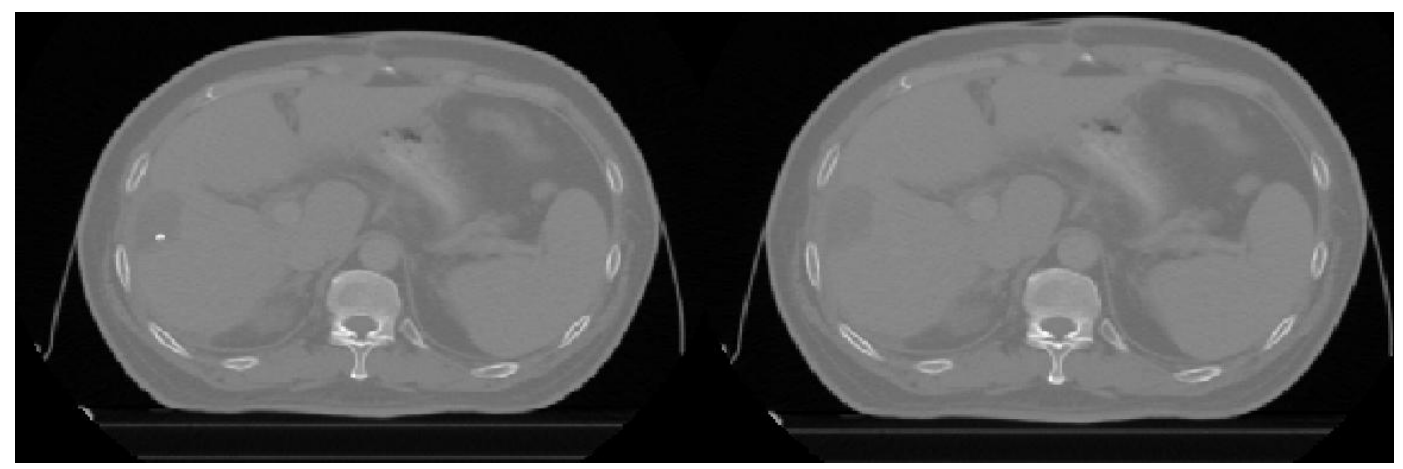

Figure 2. 4D-Avg CT images before and after the SRs digitally removed (the black circle represents the position of SRs)

\subsection{Determination of Setup Errors}

\subsubsection{CBCT- SRs Bony Matching}

Under the bony registration mode, using the minimum clip-box to encompass the SRs in CBCT image, the matching was done according to the SRs, that is to say, based on finding the maximum density in the clipbox. (Figure 3) the CBCT-SRs matching is implemented by using the cross-correlation algorithm (Hristov et al., 1996) during the acquisition of the CBCT Because of the motion of liver, the S-rings we see in the CBCT image is actually its motion coverage which border may be relatively blurred. This matching was done between the CBCT image and the 4D-Avg CT image. After image fusion and registration on XVI, the setup errors which are dependent on the patient are obtained in the in the left-right (LR), anterior-posterior (AP), and superior-inferior (SI) directions respectively.

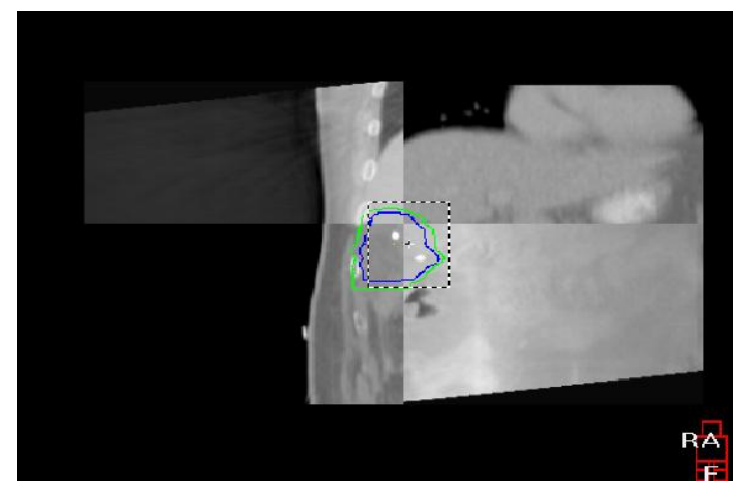

(a) coronal

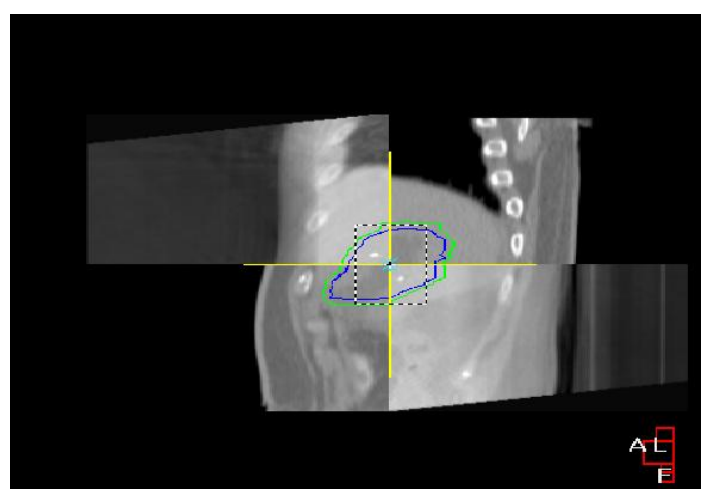

(b) sagittal

Figure 3. CBCT-SRs matching. The white dotted frame is the minimum clip-box encompassing all the SRs

\subsubsection{CBCT-ST Grey Matching}

A margin of $3 \mathrm{~mm}$ in the SI direction and $2 \mathrm{~mm}$ in the rest two directions was added to the PTV to generate a region called PTV-extended to ensure the clip-box can encompass the PTV completely during the matching course. Under the grey registration mode, using the minimum clip-box to encompass the PTV-extended, grey matching between the CBCT-ST and the reference 4D-Avg CT image without SRs were executed which are different from the manual liver-to-liver image registration conducted in other study (Hawkins et al., 2006). (Figure 4). This grey registration mode matches voxel greyscale intensity values throughout the entire clip-box, as well as the bone material. A 'grey level correlation ratio' technique is used by this algorithm. The setup errors were obtained in three cardinal directions. 


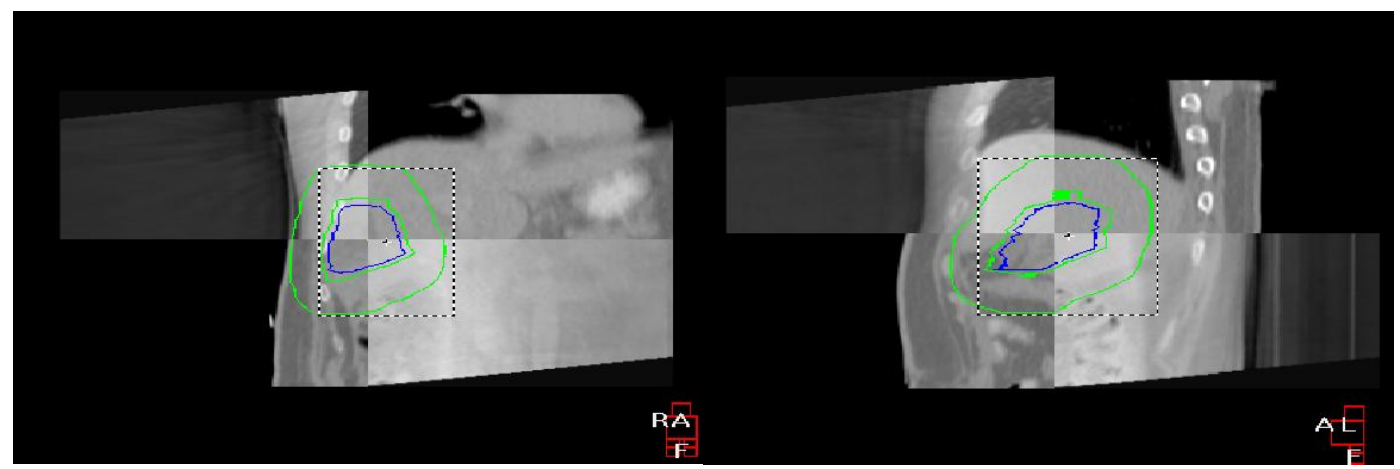

(a) coronal

(b) sagittal

Figure 4. CBCT- ST matching. The regions which are included in the solid lines are CTV, PTV and PTV-extended from inside to outside. The white dashed line was minimum clip-box which encompasses the PTV-extended

\subsection{Statistical Analysis}

The Pearson product-moment correlation coefficient calculated by Statistical Product and Service Solutions, Version 17.0 (SPSS17.0), and Excel 2007 was used to render the correlation in setup errors based on CBCT-SRs and CBCT-ST matching. The difference between these two methods was then plotted against the average couch shift as recommended by Bland and Altman (Bland et al., 1995). Provided the two methods are equivalent, the difference should be a zero mean and no significant trends. The $95 \%$ confidence interval (CI) of the error distribution is also presented.

\section{Results}

\subsection{Setup Errors}

A total of 90 cone-beam CT volumetric images with or without SRs for 10 patients were acquired. The MEAN \pm Standard Deviation (SD) of setup errors based on CBCT-SRs was $(-0.33 \pm 2.28),(-0.01 \pm 2.63)$, and $(-1.30 \pm 3.81)$ in the LR, AP, and SI directions respectively. The MEAN \pm SD of setup errors based on CBCT-ST was $(-0.54 \pm 3.05),(0 \pm 2.70)$, and $(-1.36 \pm 4.19)$ in the LR, AP, and SI directions respectively.

\subsection{Data Analysis}

Calling the perm.test function from the exactRankTests package of $\mathrm{R}$ software to Perform paired permutation test on the relationship between the two matching methods, the p-value (paired permutation test) are respectively $0.1217,0.919$ and 0.7685 in the three cardinal directions and are all greater than 0.05 .So, the null hypothesis cannot be refused, that is to say, Two methods have no significant difference. The comparison between CBCTSRs and CBCT-ST removes the temporal uncertainties because these two image modalities were taken from the same dataset. The Pearson's correlations are: $\mathrm{R}^{2}=0.718,0.785,0.724$ (Figure 5). The Bland-Altman analysis renders the $95 \% \mathrm{CI}$ of shift differences as the following: $(-2.26,2.67),(-2.86,2.84)$ and $(-3.75,3.81)$ in $\mathrm{LR}$, AP, and SI respectively (Figure 6). The percentage of agreement within $\pm 3 \mathrm{~mm}$ was $95.56 \%, 95.83 \%$ and $91.11 \%$ in LR, AP, and SI directions. The mean discrepancies of setup errors based on CBCT-SRs and CBCT-ST are $0.21 \mathrm{~mm}$ (SD 1.257), $-0.02 \mathrm{~mm}$ (SD 1.459), $0.06 \mathrm{~mm}$ (SD 1.943) in the LR, AP, and SI directions, respectively (Figure 7). 
LR

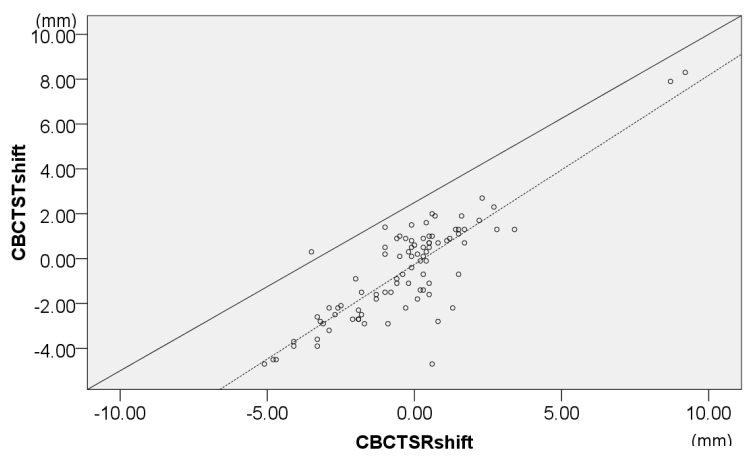

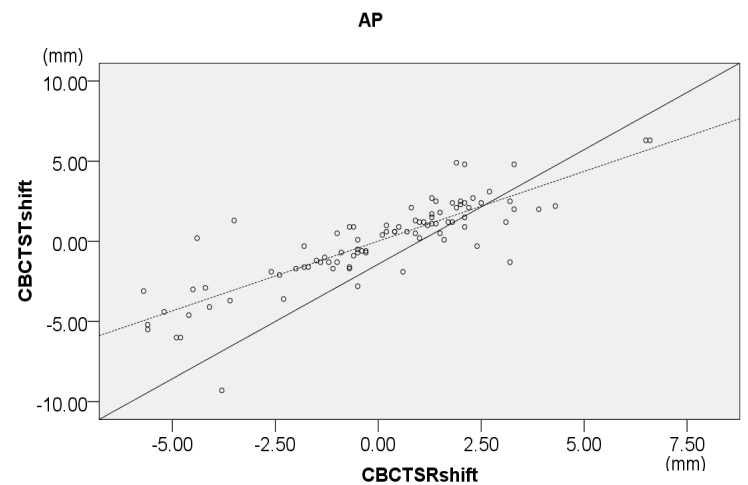

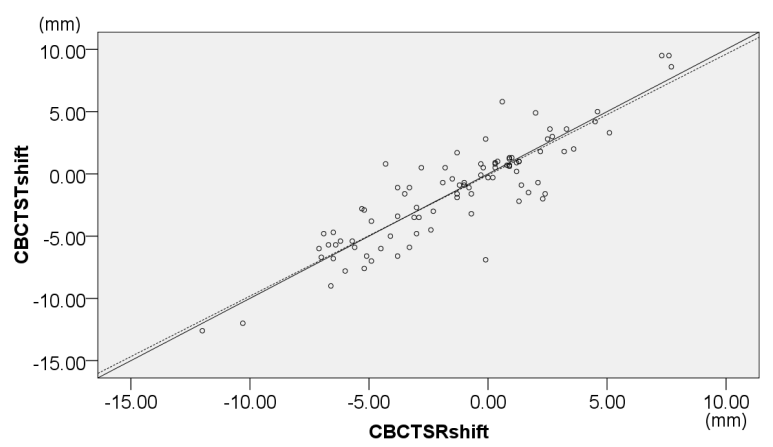

Figure 5. Two-dimensional correlations of the couch shifts based on CBCT-SRs and CBCT-ST for 10 patients in three cardinal directions
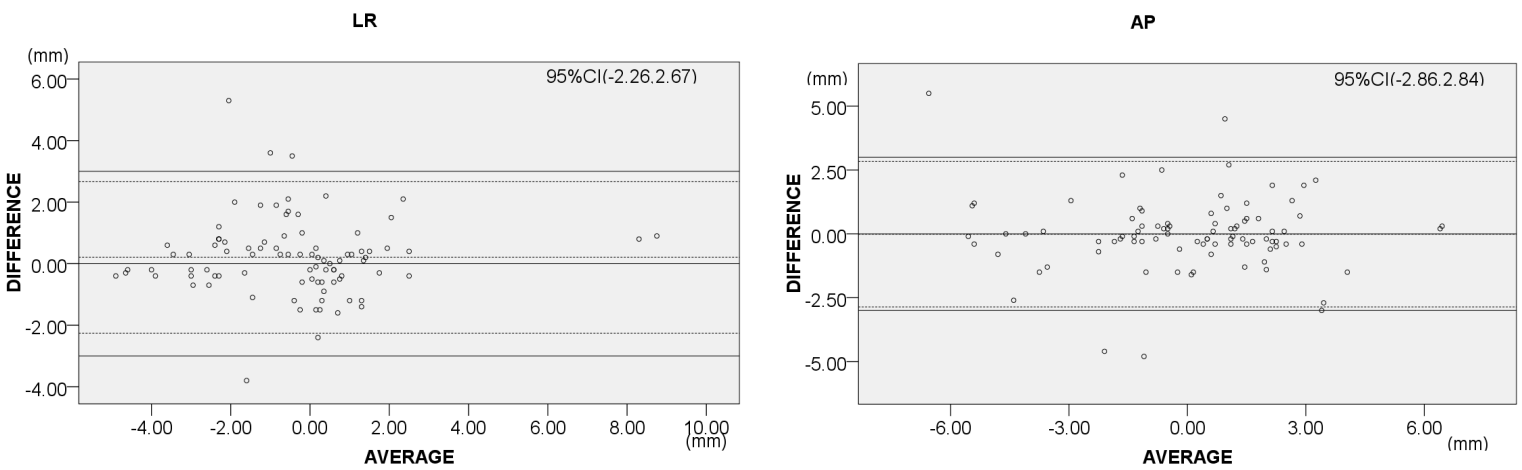

SI

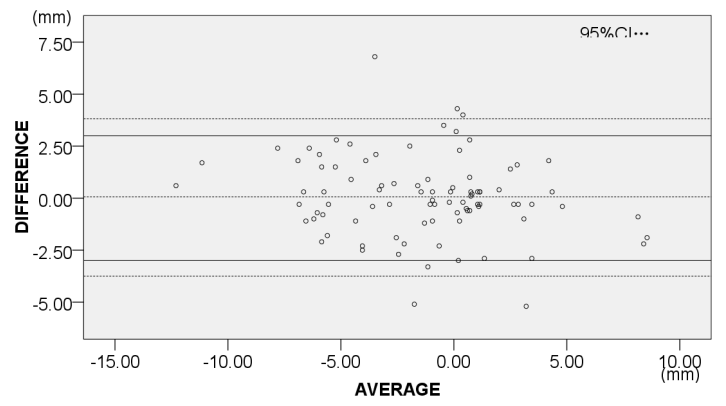

Figure 6. Bland-Altman analysis for the shifts based CBCT-ST and CBCT- SRs for 10 patients in these three cardinal directions. The current clinical action levels of $\pm 3 \mathrm{~mm}$ are drawn for reference (solid line). Letter " " represents the difference between one method and the other 

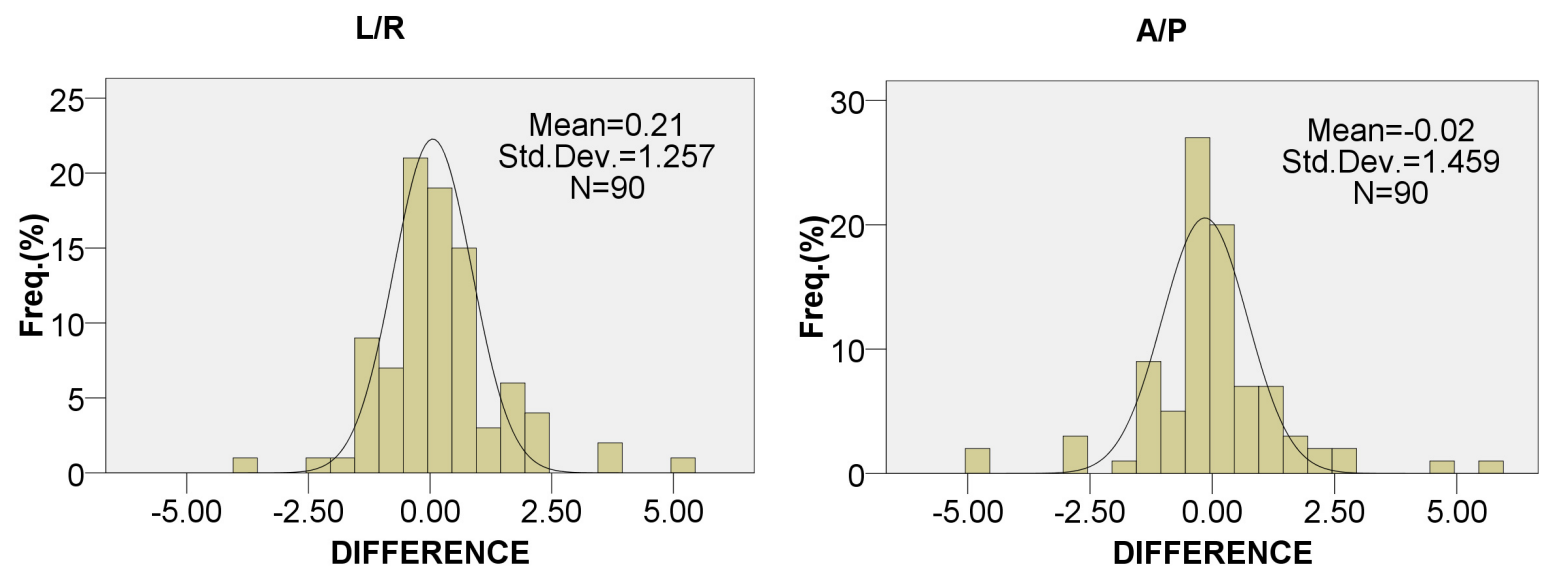

S/I

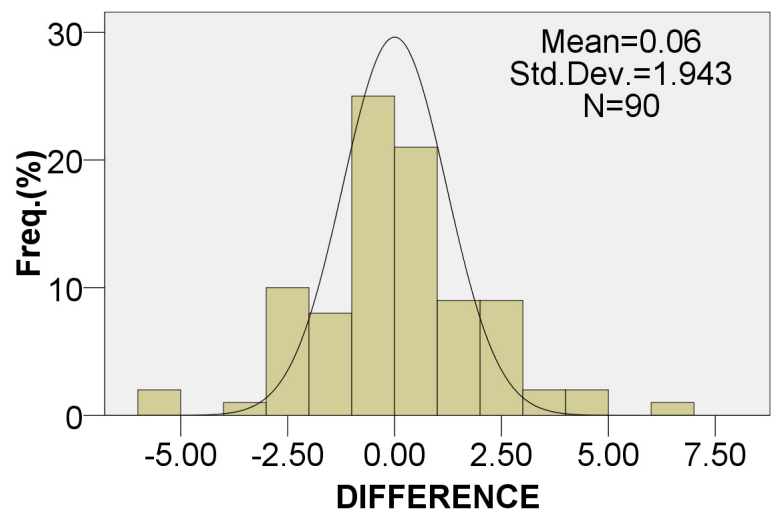

Figure 7. Plot of differences calculated between these two different methods. The histogram of the differences in the LR, AP and SI directions is presented

\section{Discussion}

According to the comparison of CBCT- SRs and CBCT-ST, The Pearson's correlations are: $\mathrm{R}^{2}=0.718,0.785$ and 0.724 in the three cardinal directions. The percentage of agreement within $\pm 3 \mathrm{~mm}$ is respectively 95.56\%, 95.83\% and 91.11\% in LR, AP, and SI directions. The high correlation and nice agreement of these two methods according to the result above and paired permutation test demonstrates that $\mathrm{CBCT}$ guidance using soft-tissue is an equivalent method used for on-line image-guided radiotherapy for liver-cancer patients when compared to CBCT guidance using fiducial markers. It is feasible to implement CBCT image guidance using soft-tissue clinically. Without substantial star artifacts induced by fiducial markers in CT and CBCT images, we can improve the accuracy of setup corrections and avoid the interference from artifacts on the dose calculation and assessment. Douglas J Moseley et al. have done the similar work to assess the accuracy of setup corrections based on CBCT-ST as compared with orthogonal megavoltage (MV) portal image-based corrections for patients undergoing external-beam radiotherapy of the prostate (Douglas et al., 2007). The correlation using soft-tissue matching was as follows: $\mathrm{R}^{2}=0.90,0.49,0.51$ in the LR, AP and SI directions. The percentage of shifts within a $\pm 3 \mathrm{~mm}$ tolerance was $99.5 \%, 70.3 \%, 78.4 \%$. They regarded it is feasible to implement online guidance based on CBCT-ST without the use of fiducial markers, provided that current PTV margins are maintained.

Although Liver-to-liver guidance allows more accurate targeting compared with $2 \mathrm{D}$ surrogates such as the diaphragm (Hawkins et al., 2006), while the limitation of such study is that the liver was used as a surrogate for the liver tumor .Furthermore, the liver tumors could not be visualized clearly. In our study, grey matching between the CBCT- ST and the reference 4D-Avg CT image without SRs were executed after using the minimum clip-box to encompass the PTV-extended. Therefore, to a great extent, this registration can be thought as tumor-to-tumor registration. 
No rotation errors were taken into consideration due to the limitations of the treatment couch, only translational errors were considered. Rotation errors cannot influence the dose delivered to the $95 \%$ of CTV markedly than the translation errors (Astreinidou et al., 2005). In this study, it hypothesized that no displacement of SRs and liver deformation took place during the treatment course so that the position of SRs can represent the liver position correctly. Influenced by the factors mentioned above, we cannot do assessment of correlation and consistence between these three methods completely.

\section{Conclusion}

This investigation has shown that CBCT-ST is feasible for daily online image guidance of the liver. Further studies to explore and develop software for automatic removing the SRs without any of them being missed are expected.

\section{Acknowledgements}

This research work was funded by National Natural Science Foundation of China (Grant No. 10975187).

\section{References}

Astreinidou E., Bel-Aa, Raajmakers, C. P., Terhaard, C. H., \& Lagendijk, J. J. (2005). Adequat margins for random setup uncertainties in head-and-neck IMRT. Int. J. Radiat. Oncol. Biol. Phys., 61, 938-944.

Bland, J. M., \& Altman, D. G. (1995). Comparing methods of measurement: Why plotting difference against standard method is misleading. Lancet, 346, 1085-1087.

Moseley, D. J., White, E. A., Wiltshire, K. L., Rosewall, T., Sharpe, M. B., Siewerdsen, J. H., ... Jaffray, D. A. (2007). Comparison of localization performance with implanted fiducial markers and cone-beam computed tomography for on-line image-guided radiotherapy of the prostate. Int. J. Radiat. Oncol. Biol. Phys., 67, 942-953.

Feldkamp, L. A., Davis, L. C., \& Kreiss, J. W. (1984). Practical cone beam algorithm. J. Opt. Soc. Am., 612-9.

Hawkins, M. A., Brock, K. K., Eccles, C., Moseley, D., Jaffray, D., \& Dawson, L. A. (2006). Assessment of residual error in liver position using $\mathrm{kV}$ cone-beam computed tomography for liver cancerhigh-precision radiation therapy. Int. J. Radiat. Oncol. Biol. Phys., 66(2), 610-9.

Hristov, D. H., \& Fallone, B. G. (1996). A grey-level image alignment algorithm for registration of portal images and digitally reconstructed radiographs. Med. Phys., 23, 75-84.

Jaffray, D. A., Siewerdsen, J. H., Wong, J. W., \& Martinez, A. A. (2002). Flat-panel conebeam computed tomography for image-guided radiation therapy. Int. J. Radiat. Oncol. Biol. Phys., 53, 1337-1349.

Owen, R., Kron, T., Foroudi, F., Cox, J., Zhu, L., Cramb, J., ... Duchesne, G. (2008). The detectability and localization accuracy of implanted fiducial markers determined on in-room computerized tomography (CT) and electronic portal images (EPI). Med. Dosim., 33, 226-233. http://dx.doi.org/10.1016/j.meddos.2007.12.002.

Sharpe, M. B., Moseley, D. J., Purdie, T. G., Islam, M., Siewerdsen, J. H., \& Jaffray, D. A. (2006). The stability of mechanical calibration for a $\mathrm{kV}$ cone beam computed tomography system integrated with linear accelerator. Med. Phys., 33, 136-144.

Wunderink, W., Mendez Romero, A., Seppenwoolde, Y., de Boer, H., Levendag, P., \& Heijmen, B. (2010). Potentials and limitations of guiding liver stereotactic body radiation therapy set-up on liver-implanted fiducial markers. Int. J. Radiat. Oncol. Biol. Phys., 77, 1573-1583. http://dx.doi.org/10.1016/j.ijrobp.2009.10.040

Wunderink, W., Mendez-Romero, A., de Kruijf, W., de Boer, H., Levendag, P., \& Heijmen, B. (2008). Reduction of respiratory liver tumor motion by abdominal compression in stereotactic body frame, analyzed by tracking fiducial markers implanted in liver. Int. J. Radiat. Oncol. Biol. Phys., 71, 907-915. http://dx.doi.org/10.1016/j.ijrobp.2008.03.010

\section{Copyrights}

Copyright for this article is retained by the author(s), with first publication rights granted to the journal.

This is an open-access article distributed under the terms and conditions of the Creative Commons Attribution license (http://creativecommons.org/licenses/by/3.0/). 